CLAWAR 2020: $23^{\text {rd }}$ International Conference on Climbing and Walking Robots and the Support

Technologies for Mobile Machines,

Moscow, Russian Federation, 24-26 August 2020.

https://doi.org/10.13180/clawar.2020.24-26.08.64

\title{
A ROBOT DESIGN FOR WIND GENERATOR SUPPORT STRUCTURE INSPECTION
}

\author{
SHYAMAL C. MONDAL and PATRICIO L. C. MARQUEZ \\ London South Bank University \\ School of Engineering \\ 103 Borough Road, London SE1 OAA, UK
}

\begin{abstract}
In recent time, the development of wind tower inspection has been very crucial for the overall performance of the wind turbine. In order to maintain, monitor and determine the life span of the tower, an investigation of robot design is discussed. It presents how to design and construct a robot that can climb the tower and rotate $360^{\circ}$. A ring system which is in a circular shape robot is designed that allows the device to fit in the structure of the wind generator tower. The rotational module is designed to allow the wheels to rotate and be able to go in a circular motion. Also it is designed with a suspension that allows the robot to go through any obstacle. This paper also presents the FEA spring stress analysis and Simulink control system model to find the optimal parameters that are required for the wind tower climbing robot.
\end{abstract}

\section{Introduction}

The industrialised nations are trying to legislate for carbon zero emission by 2050 . This pledge can be achieved by changing the energy production from unrenewable to renewable energy such as wind power or have the capabilities to remove carbon particles from the atmosphere through a process called carbon offsetting. The main concern with the overall operation of the wind tower is that it is too expensive to inspect and to maintain. Also, it is dangerous or hazardous for people who carry inspection on the tower [1].

The current method to inspect the wind tower structure that is mostly used is scaffolding that allows locating of any damages in the tower, generator and blades. It is not only dangerous for humans but also it requires a substantial cost to the company. Therefore, an automated climbing robot would an advantages for the inspection of the wind tower. The common ways that the robot is used for climbing the wind tower of a structure are the following: the adhesion methods which use magnets in the wheel that will provide a strong adhesion force to the tower and spring loaded adhesion method [2,3]. Another method is electro-adhesion. Electro-adhesion uses electrodes to be able to create an electrostatic force of attraction from the robot to the surface.

The magnetic method that is commonly used to inspect, this is utilised in the wheels as this provides a strong adhesion force to the surface so this will avoid any loss of tractive force. The orientation of the magnets that are on the wheel will be about the same distance from the surface, which it will determine the gap between the magnets [4]. This method is fast and very reliable plus it has a strong adhesion force. However, the problem is that it can only be used on the surface that has a strong magnetic force, and this method is not very efficient as it wastes energy.

The suction adhesion method is employed in various applications like inspecting tall buildings, such as cleaning windows. So, in order, the robot stays on a vertical wall, the need for two mechanisms active and passive adhesion. The active adhesion uses the negative pressure or electrostatic to exert strong adhesion force in which it will allow to control the attachment and detachment, so to do this it requires like a vacuum pump. However, the passive adhesion mechanism can have a large adhesion force, and it could be used without a power supply, and it will not be necessary an actuator to change the attachment and detachment of the robot [5]. 
However, having looked through projects, this method will not be useful on all surfaces especially in surfaces that are bulky or surfaces that are not smooth or clean.

The electro-adhesion is another technique in which it uses electrodes to be able to create electrostatic force attraction from the device to the surface; this gives an excellent adhesion in most materials like wood, concrete and steel [6]. However, this process fails when there is a surface that has moisture and only moves in one direction. On the other hand, electro-adhesion can make a device lighter, and it will allow using very low energy.

Dry adhesion method was inspired by the creature that is the geckos, or another way that can be called is the Van Der Waals force. This technique can generate forces between the surface and the fibril that is usually attached to a climbing robot. This consists of three stages in which the first one is to connected to the surface, then is the preloading in which there is an increase in the adhesion force, and the last one is peeling which removes the adhesive from the surface, so the robot will be able to move. This method is similar to the gecko feet [7]. Some climbing robots do not use much energy for the adhesions which show a great potential in the industry. However, many studies state that the moisture and the dust on the surface could reduce the adhesion mechanism.

The wheeled method uses magnetic wheels like the adhesion method. However, there is a technique that is different from the usual methods employed to inspect cylindrical structures. These are a prototype that was built in a project for London South Bank University which consists of three modules and joined in a triangular structure in which the robot can climb along the pipe, and the wheels can rotate 90 degrees. This can be done by using spring forces to grip around the tower and be able to climb[8].

The legged method is commonly used. This type of robot is designed depending on the task that needs it. It has several controllable joints that allow a multi-degree of freedom for the mobility. This kind of robot will have some vacuum cups that are located at each foot of the device. So depending on how many legs the robot has, the legs are separated into two groups. The first pair of legs will be working on attaching the legs to the surface, and the other group will be moving to the desired position and adhere to the surface [9]. This type of robot has a multi-degree of freedom that is suitable for uneven structures. However, the Locomotion consumes a significant amount of energy, and the mobility of the robot is slow as it does not have continuous movement.

The tracked method is mostly used in applications such as inspection and maintenance, this type of robot is relatively faster and less complex to build. Some robots also use the magnetic adhesion method that has modules to move the robot. Others have tracked belts with some magnets in the belt, so this will allow magnetic adhesion with the surface, but it consumes more energy than the wheeled robots. The prototype that will be developed needs to climb the whole wind turbine structure. The average height of a wind turbine support structure is around $150 \mathrm{~m}$ depending on the area and the country regulations; it is usually made of steel [10]. The prototype is required to climb the structure and be able to rotate 360 degrees around; this will allow the operator to have a better view of the structure and the blades of the wind turbines.

The design of the robot needs to handle the load as this will allow the operator to add any sensor to inspect the structure. Also can be adjustable to add a Cartesian scanning arm in which it will allow the robot to climb to the top of the tower and be able to scan for any damages that could be found in the blades of the turbines [11].

\section{Analysis of the prototype design of the robot}

The robot needs to be able to go up and down in a considerable speed in order to reduce inspection time plus it needs to rotate 360 degrees. The structure of the design needs to adapt to the tower of the wind turbine. The structural design of the robot needs to be light and be able to adapt to any environmental conditions of the wind turbine structure. 
The Figure 2.1(a-c) below shows a 3D design of robot in AutoCAD. From the design it can be seen that the robot has three rings that are fixed to each other, and each ring is distributed at 120 degrees to each other in a circular way.

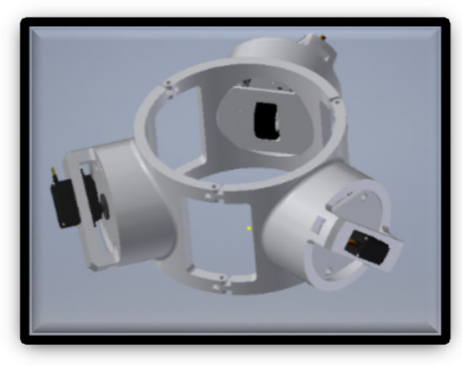

(a)

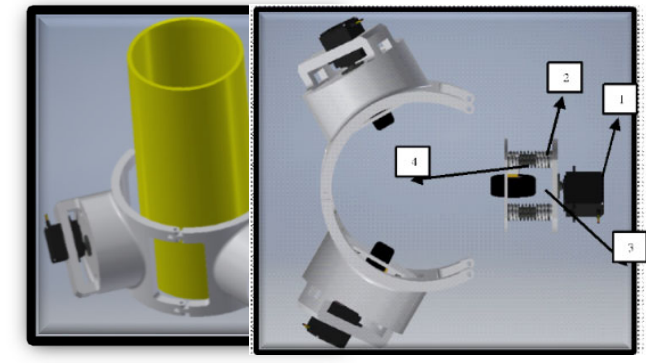

(c)

Figure $2.1(\mathrm{a}-\mathrm{c})$ : Design of the robot in AutoCAD Inventor

In Figure 2.1(c), it can be seen that it has three servo motors (1), each of the servos is attached to the base of the rotational module. The use of springs (2) will help the robot to adjust to a tower and also it will help to overcome any obstacle that can be found in the tower. The parts (3) and (4) will be connected to the micro geared motor which will move the wheel in any direction, like up and down or in a circular motion.

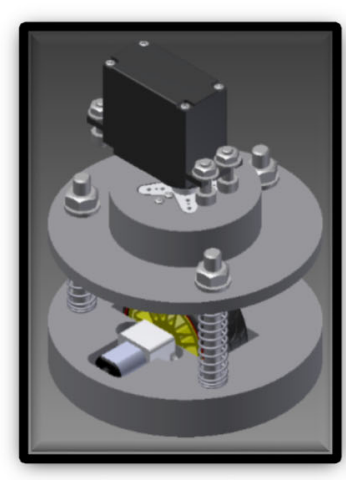

( a )

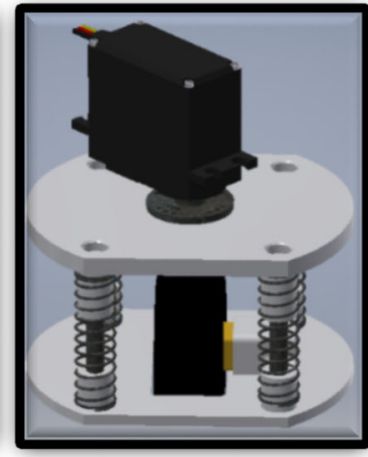

(b)

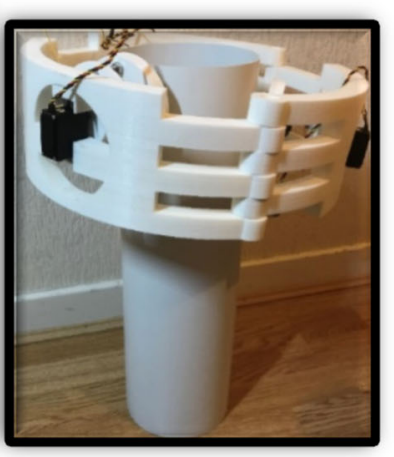

(c)

Figure $2.2(\mathrm{a}-\mathrm{b})$ : View of the rotation module ; $(\mathrm{c})$ : A prototype module

The rotational module as shown in Figure $2.2(\mathrm{a}-\mathrm{b})$ that will allow the robot to rotate 360 degrees around the tower. The servo motor is mounted in a suspension structure, as the servos are in a central position ( 0 degrees). This will allow the robot to move up and down the tower. However, when the servos rotate to the desired angle (90 degrees), this will enable the robot to go in a circular motion, which will meet the requirements for this project.

The prototype module of the robot is shown Figure 2.2 (c). As each module is at 120 degrees from each other, this allows the robot to hold firmly onto any surface. The wheels that are fix every 120 degrees in the form of an equilateral triangle gives the robot stability when it is climbing, as the mass is distributed to the centre of the robot, which allows more stability.

\section{Spring Analysis}

The spring will help the robot to adjust to a tower to overcome any obstacle that can be found in the tower, so it is necessary to make calculations to find the suitable spring for the robot. 
The calculation of the spring preload to hold the weight of the assembly robot that is approximately of $1.55 \mathrm{~kg}$ and with a safety factor of 2.5 . $W=m * g ; W=1.55 \mathrm{~kg} *$ $9.81 \mathrm{~m} / \mathrm{s}^{2} ; W=15.21 \mathrm{~N}$

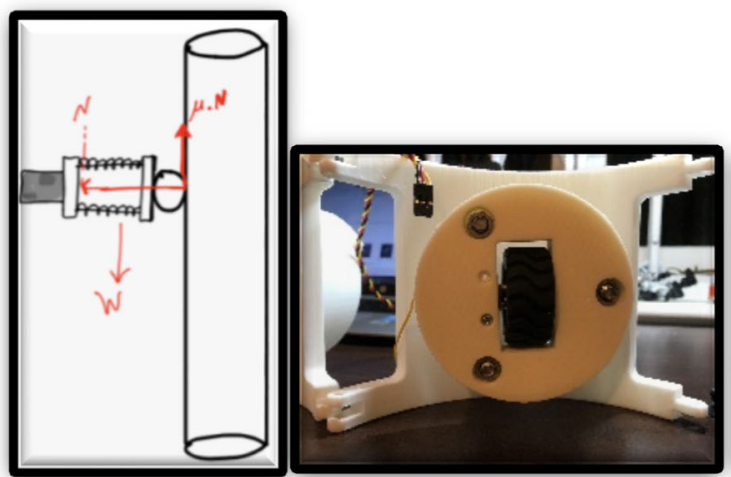

(a)
( b )

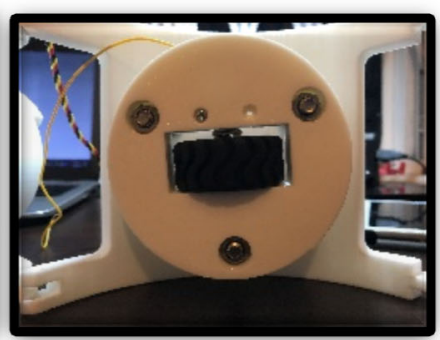

(c)

Figure 3.1 (a): Forces on the spring ; (b): Servo translational movement; (c): Servo rotational movement

Adding a safety factor of 2.5 for the mass of the assembly, the resulting $W$ is $15.21 * 2.5=$ $38.03 \mathrm{~N}$.

force that needs to be restrained by a lateral force from the spring - that force is $\mathrm{N}$ as shown in Figure 3.1 (a).

So $\mu * N \geq w / 3$ assuming that $\mu=0.18$ the coefficient of friction and $w / 3$ is to take into account that $1 / 3$ of the weight, to be able to calculate the lateral force for one set of the wheels. Therefore, $0.18 * N=12.7 ; N=70.6 N$.

Each wheel of the robot needs to exert $70.6 \mathrm{~N}$ through the springs, as the springs are parallel, the force is divided equally between them, and each must be preloaded with around $24 \mathrm{~N}$ to hold the weight of the device.

Figure 3.1 (b \&c) shows the wheel position for the translational movement and rotational movement for the robot.

\section{Simulation of the robot control}

The control simulation was modelled in Simulink software to monitor the expected output parameters that was required for the robot as shown in Figure 4.1

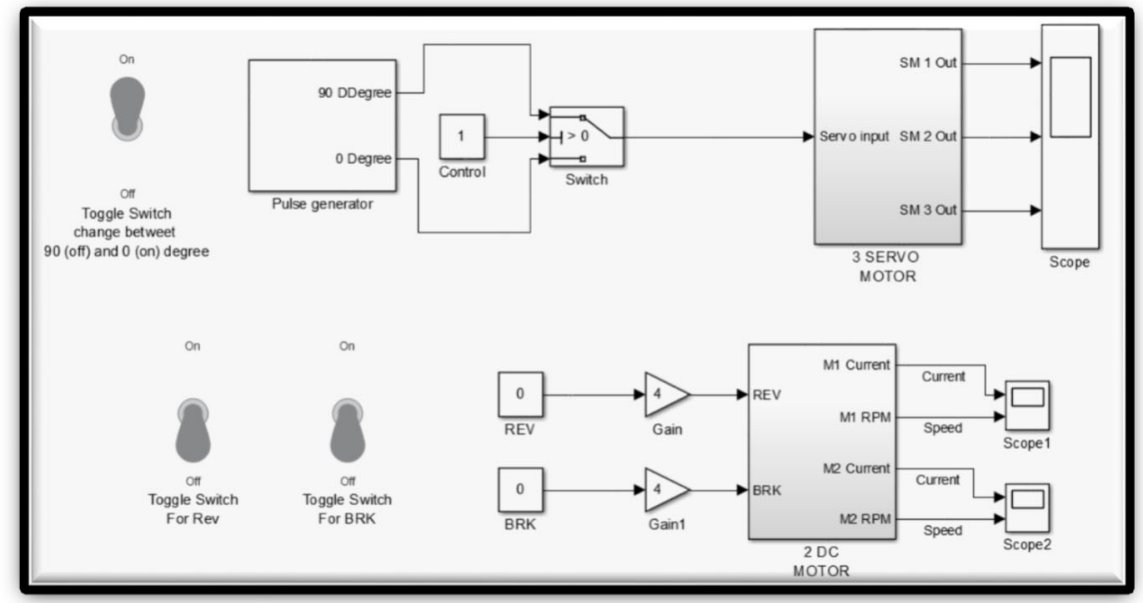

Figure 4.1 System to control the robot 


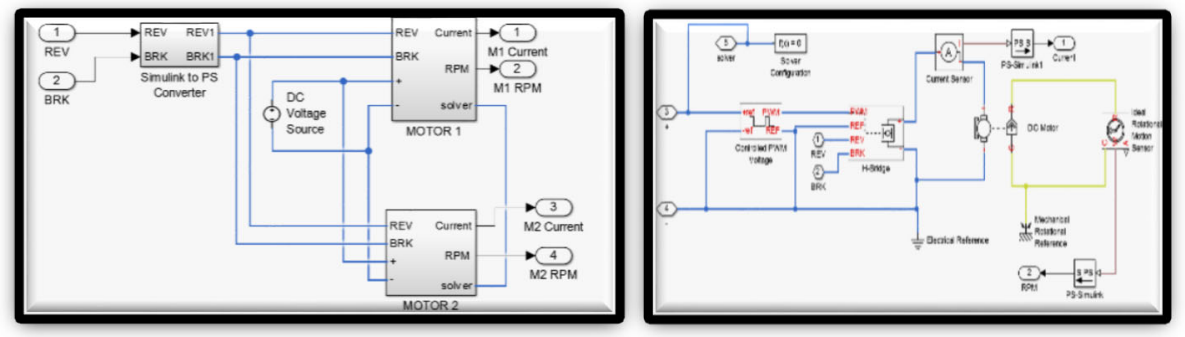

(a)

(b)

Figure 4.2 (a): DC motor connection ; (b): Block diagram of the DC motor

The Figure 4.2 (a) shows the connection of the DC motors. They are both connected to the same supply and control signal. The two DC motors are to behave in a similar way. The DC motor block has four inputs and two Outputs. The two inputs supply a rail, while the other two are for reversing the direction of the motor and breaking it. The output of the motor is current and the speed of the motor. During forward motion, the speed is positive and when reversing the speed is negative. The DC motor will only reverse or break if the control signal is greater than the threshold voltage of 2.5. Figure 4.2 (b) shows inside the block diagram of the DC motor. The block diagram of the DC motor comprises of a Controlled PWM voltage, an H-Bridge, Current Sensor, DC motor and Rotational motion sensor.

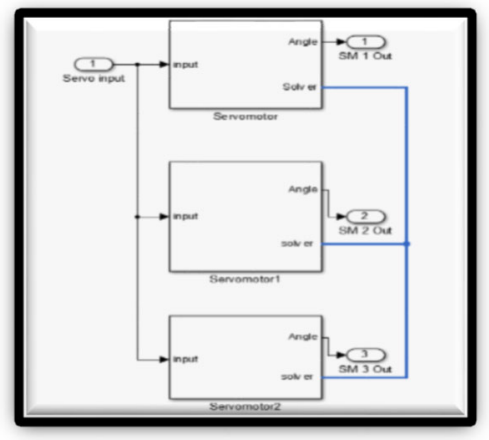

(a)

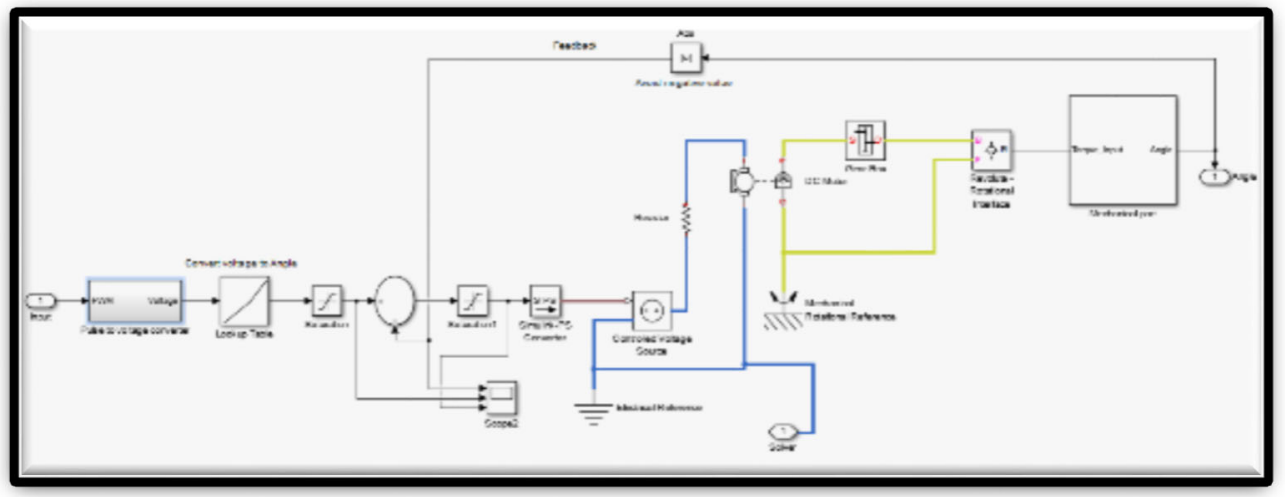

( b )

Figure 4.3 ( $\mathrm{a} \& \mathrm{~b}$ ): Simulink model of the servo motor 
The model of the servo motor is shown in Figure 4.3 (a). The three servo motors have the same inputs, and their outputs are the same. Figure 4.3(b) shows the model of the servo motor which is comprised of two parts: electrical and mechanical. The electrical part is comprised of a pulse to voltage converter which converts the pulse signal to an analogue voltage. This voltage then converts the angle using the lookup table. A saturation block is used to make sure when converting, that the angle does not exceed 180 degree, which is the maximum the servo motor can rotate. The angle is then fed into a controlled voltage source to be applied to the DC motor. The DC motor is connected to a gear of ratio 5.

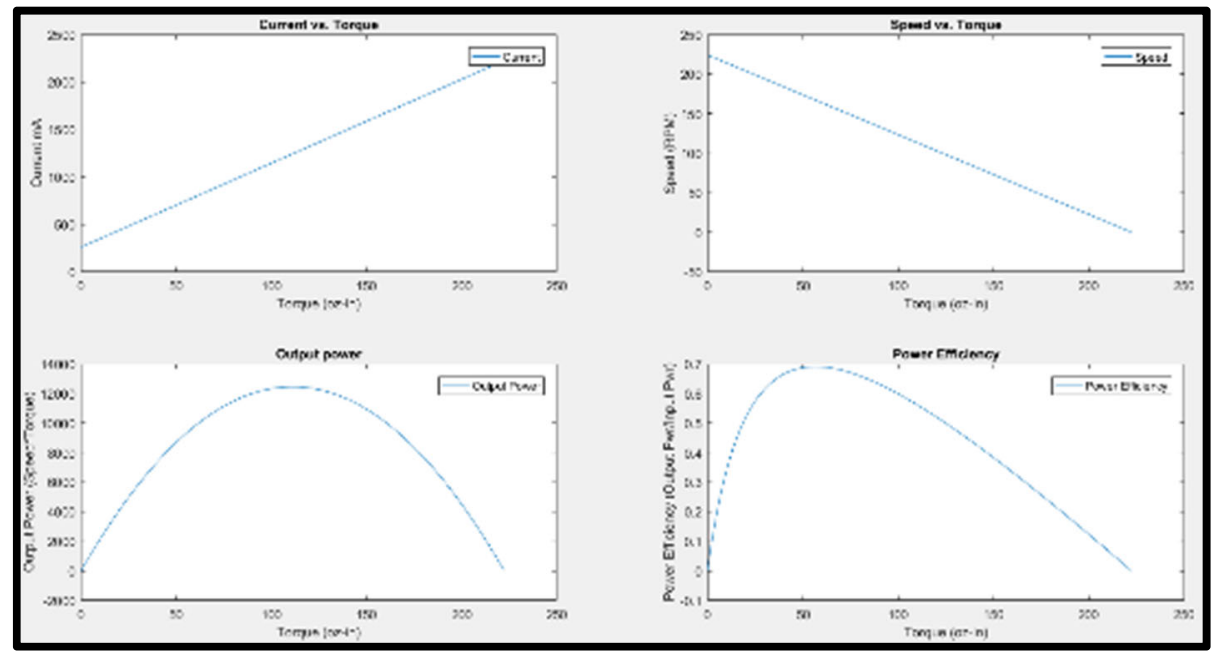

( a )

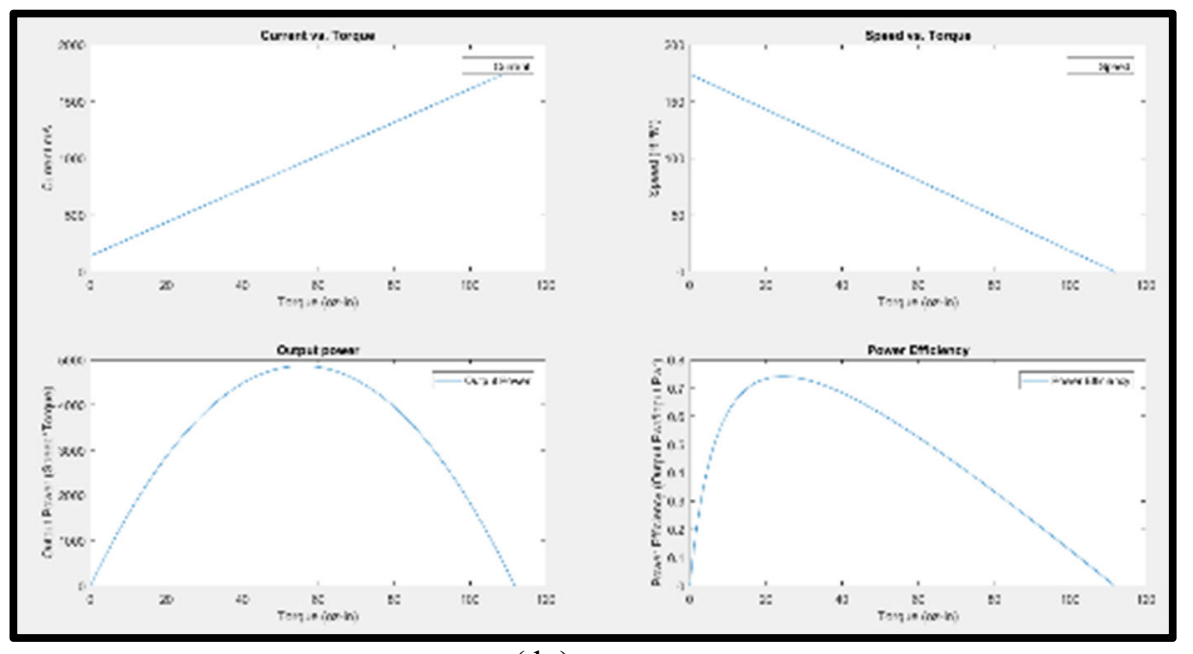

( b )

Figure 4.4 (a): Simulation Torque analysis results at 9V; (b) : Simulation Torque analysis results at $18 \mathrm{~V}$

Figure 4.4 (a \&b) shows the simulation results of torque against current, speed, output power and power efficiency for the two input voltages of $9 \mathrm{~V}$ and $18 \mathrm{~V}$. The torque is proportional to current and inversely proportional to speed. The power efficiency increases up to a certain value of torque and then decreases linearly. 


\section{Stress Analysis}

In the development of the robot in which a 3D model is created in AutoCAD as shown in Figure 7.1 (a-b), it allows to make an analysis of the stress that will be placed in the structure when the robot is moving.

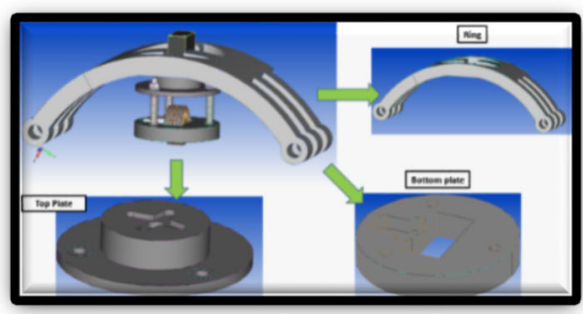

(a)

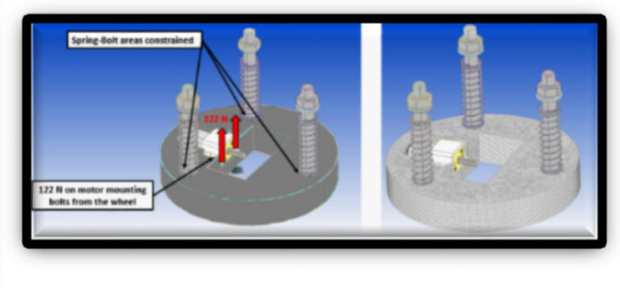

(b)

Figure 5.1 (a): All the parts that will be analysed; (b): Force applied

Figure 5.1 (b) shows the force that was applied to the bolts of the bracket that fix the micro gear motor to the base, as this part will hold an important job, as it has to support all of the stress and force that will be applied when the robot is moving.

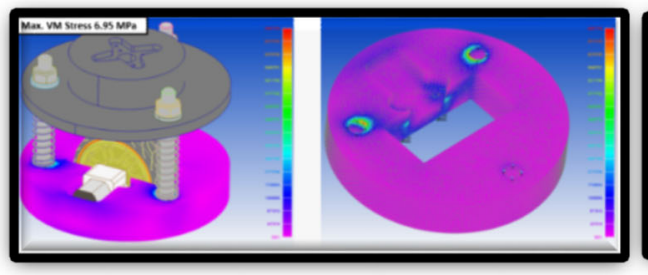

( a )

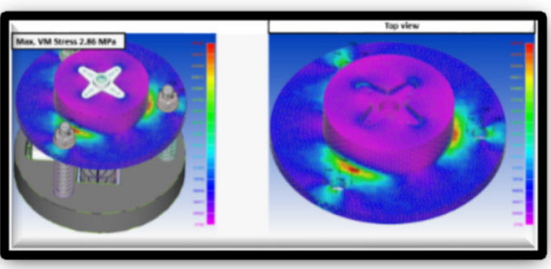

(b)

Figure 5.2 (a): Stress on the Plate holding the servo motor ; (b): Stress on the upper plate

The Figure 5.2 (a) shows that the maximum stress that was applied is around $6.95 \mathrm{MPa}$. It indicates that the materials used for the prototype will be able to cope with the stress that will be generated, as the ABS Plastic Yield Stress is around $44 \mathrm{MPa}$, which means that there will be a significant margin of safety results from the analysis.

Figure 5.2 (b) shows the stress that will be available at the base of the servo motor, in which the servo hub will be attached to the base, as this will allow the rotation of the module. The upper plate has a maximum stress of $2.86 \mathrm{MPa}$ at the top of the plate, which has a significant margin of safety as the ABS Plastic Yield Stress is around $44 \mathrm{MPa}$.

\section{Conclusions}

A prototype laboratory based climbing robot was developed in order to test on the laboratory based vertical tower. The protype robot has not used any specific scale ratio and dimensions that can be used for the real application. The robot was able to climb up and down on the tower and rotate 360 degrees around the tower. A circular ring shape designed robot suited the structure of the wind generator tower, and was able to move in a stable manner

However, there was one main issue with the robot, as the acceleration torque required for the robot to climb was much higher than anticipated, and so higher torque motors were needed. On the other hand, the robot seems to have no difficulty providing enough adhesive force, this was developed by the spring mechanism. 
There are some improvements that have to be made in order to transform the robot to real industrial use. A differential drive can be implemented by introducing a tracked locomotion to the robot for better manoeuvrability and traction, and an embedded pressure sensor can be implemented into the electrical system to monitor the payload during real life operation. This is essential as the payload increases greatly due to aerodynamic resistance.

\section{References}

[1] Anne Jungert "Damage Detection in Wind Turbine Blades using two Different Acoustic Techniques", The e-Journal of Nondestructive Testing (December, 2008),

[2] Raju D.Dethe ,Dr. S.B. Jaju, "Developments in Wall Climbing Robots: A Review”, International Journal of Engineering Research and General Science Volume 2, Issue 3, (AprilMay 2014),

[3] Joseph L. Jones, Anita M. Flynn, Mechanics, Mobile Robots, Inspiration to Implementation, p139, (Accessed on 30/10/2015)

[4] Weimin Shen, Jason Gu, "Permanent Magnetic System Design for the Wall-climbing Robot", Proceedings of the IEEE International Conference on Mechatronics \& Automation Niagara Falls, Canada, (July

[5] Kang Liu, Wenzeng Zhang, "Symmetrically centralized magnetic-wheel unit for wallclimbing robots", Proceedings of the $13^{\text {th }}$ IASTED International Conference ROBOTICS AND APPLICATIONS, (August 29-31, 2007),

[6] Minghui Wu, Gen Pan, Tao Zhang, Shanben Chen, Fu Zhuang, Zhao Yan-zheng, "Design and Optimal Research of a Non-Contact Adjustable Magnetic Adheson Mechanism for a Wall-

Climbing Welding Robot", International Journal of Advanced Robotic Systems, (October, 2012),

[8] R.C.Hibbeler, Engineering Mechanics Statics, Twelfth Edition in SI Units, Chapter 5

[9] Waldron, K. et al. Climbing ring robot for inspection of offshore wind turbines. Industrial Robot: An International Journal, 2009, 36 (4), pp. 326-330.

[10] J. Shang, B. Bridge, T. Sattar, S. Mondal and A. Brenner "Development of a climbing robot for the NDT of long weld lines", Industrial Robot: An international Journal, vol. 35, Issue 3, May (2008).

[11] B. Bridge, T. Sattar, and Khalid, A., "On the Design of Multi-Task Compact, Climbing Robotic NDT Systems for Remote Operation on Large Surfaces In Hazardous Environments, Nondestructive Testing and Evaluation", Vol. 13, pp. 85-111, (1997). 